${ }^{1}$ Fundación Arriarán. Santiago, Chile.

${ }^{2}$ Hospital Clínico San Borja Arriarán. Santiago, Chile.

${ }^{3}$ Facultad de Medicina,

Universidad de Chile. Santiago, Chile.

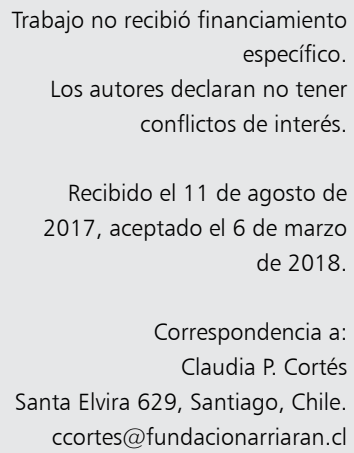

1 1 advenimiento de la terapia antirretroviral moderna (TAR) ha llevado a una enorme reducción de la mortalidad y morbilidad en pacientes con infección por $\mathrm{VIH}^{1-3}$. No obstante, persiste una considerable tasa de abandono a controles médicos e irregular adherencia al tratamiento ${ }^{4-7}$. Se observa un progresivo aumento de

\section{Evolución de mortalidad, abandono, traslado y retención a corto, mediano y largo plazo en pacientes con infección por VIH: Fundación Arriarán 1990-2015}

\author{
CLAUDIA P. CORTÉS ${ }^{1,3}$, DANAE LIZANA ${ }^{1,2}$, \\ REBECA NORTHLAND ${ }^{1,2,3}$, MARCELO WOLFF R. ${ }^{1,2,3}$
}

\section{Evolution of mortality, retention and loss to follow up of HIV infected subjects followed in a special clinic}

Background: The HIV epidemic reached Chile in late 1980s and as an early response, AIDS care centers were organized. Fundación Arriarán (FA) was the first center. Free antiretroviral therapy (ART) was later provided with progressive coverage and complexity over the years. Aim: To quantify evolution of mortality, retention and loss to follow up (LTFU) over 25 years according to different periods of access to ART, from no availability to full coverage with current drugs at FA center. Material and Methods: Retrospective analysis of FA database of 5,080 adults admitted between 1990 and 2014. The sample was distributed in 7 groups: A: no ART (1990-92), B: monotherapy, C: dual therapy, D: dual/triple ART, E: early triple therapy with incomplete coverage, $F$ same as E but with complete coverage and G: contemporary ART (2008-14). Mortality, retention and LTFU were evaluated at 1, 3, 5, 7 and 10 years and at 31/12/2015. Results: Mortality varied from $40 \%$ to $2 \%$, and $62 \%$ to $7 \%$ at 1 and 5 years, for groups $A$ and $G$ respectively; from $71 \%$ to $16 \%$ at 10 years for groups $A$ and E, respectively. Retention at 5 years were 28\%, 23\%, 39\%, 62\%, $75 \%, 75 \%$ and $77 \%$ for groups A to G, respectively. LTFU was $10 \%, 19 \%, 15 \%$, $17 \%, 9 \% 12 \%$ and $10 \%$ at 5 years for same groups, respectively. At 12/31/2015 $22 \%$ of patients had died, $11 \%$ were LTFU, $60 \%$ were retained in care and $6 \%$ had been transferred. Conclusions: There is a marked reduction in mortality and increase in retention of HIV patients' concomitant to expanded access to modern therapy, although LTFU remains a problem.

(Rev Med Chile 2018; 146: 290-299)

Key words: Cohort Studies; HIV; Lost to Follow-Up; Mortality; Survival.

la sobrevida y calidad de vida desde el inicio de la TAR al ir aumentando el acceso a terapia y su monitoreo, al desarrollo de nuevas drogas antirretrovirales y actualizadas estrategias de tratamiento. Estas nuevas recomendaciones se caracterizan por un inicio cada vez más precoz de la terapia, el uso de medicamentos con menos toxicidades 
e interacciones y una mejor posología, llevando a una mejoría en la adherencia al tratamiento y a mejores tasas de éxito clínico, inmunológico y virológico, así como a menor transmisibilidad viral $^{8,9}$. En este último aspecto se ha consolidado el concepto de "tratamiento como prevención" de nuevas infecciones ${ }^{10}$.

Desde el inicio de la epidemia de VIH han ido ocurriendo diferentes hitos a nivel internacional y nacional en el manejo de la enfermedad que han optimizado los tratamientos y mejorado el acceso, contribuyendo a un éxito terapéutico pocas veces visto en enfermedades crónicas. A nivel nacional estos hitos comienzan con la creación de la Comisión Nacional del SIDA (CONASIDA) en $1990^{11}$, iniciándose un período de políticas públicas destinadas a mejorar el manejo de la infección por VIH. La primera medida fue la creación de programas y centros de atención VIH para la prevención y control de la enfermedad, distribuidos en todo el territorio, seguida de una lenta pero progresiva disponibilidad de medicamentos y monitoreo del tratamiento, hasta llegar a disponer de cobertura garantizada nacional tanto a nivel público como privado con antirretrovirales modernos y técnicas de monitoreo de alto nivel. En 1991 se publica la ley 19.779, conocida como ley del SIDA, que aporta un marco jurídico a las intervenciones relacionas con la enfermedad. El 2005 se implementa la incorporación de la infección por VIH dentro de las patologías con garantía explícitas de tratamiento, oportunidad y protección financiera $(\mathrm{GES})^{13}$. Paralelamente, las guías clínicas terapéuticas fueron gradualmente promoviendo el inicio de tratamiento en etapas más tempranas de la enfermedad ${ }^{14-17} \mathrm{y}$ con fármacos con mejores perfiles de seguridad y eficacia (Figura 1).

El centro de atención de VIH del Servicio de

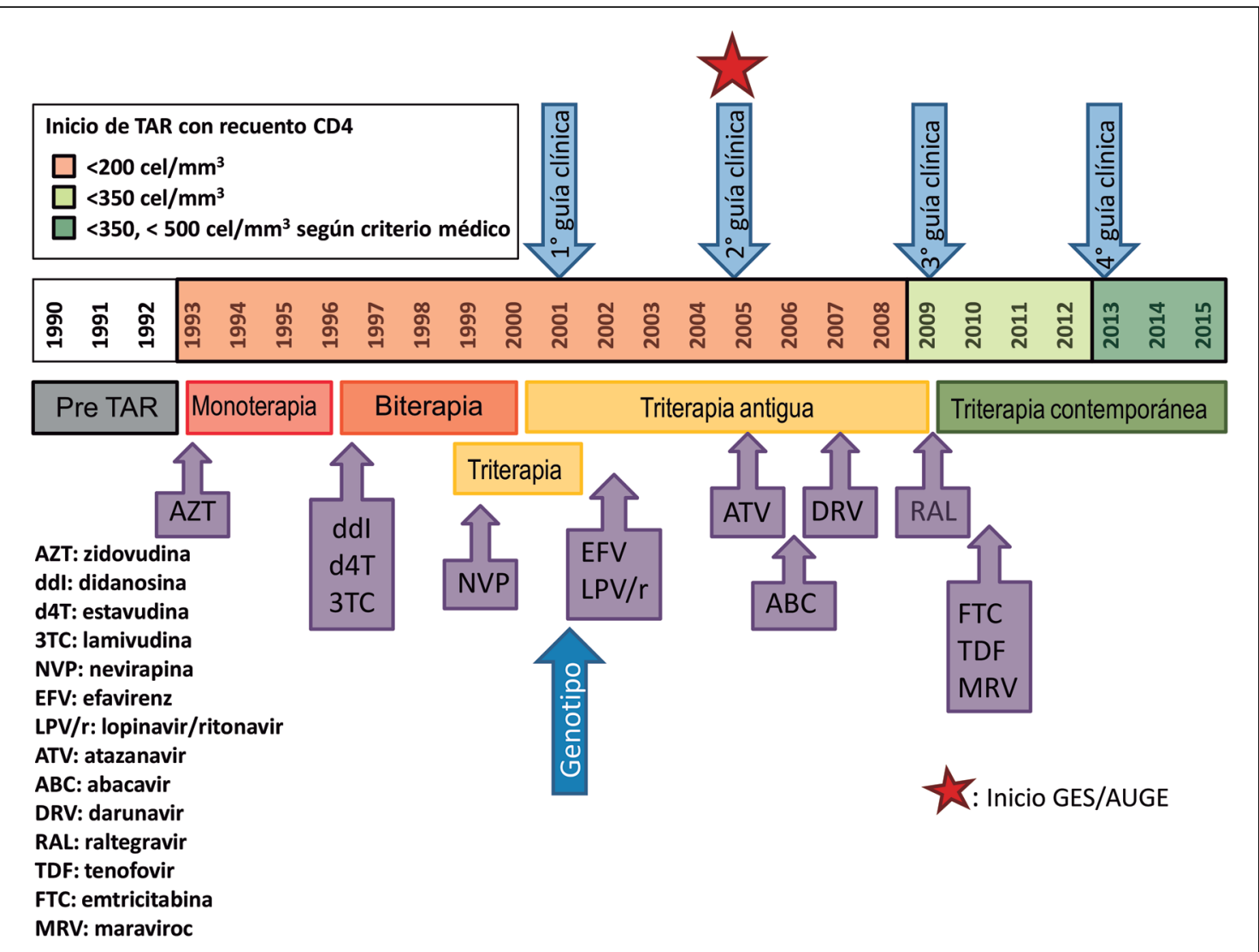

Figura 1. Línea del tiempo en la evolución del manejo de la infección por VIH en Chile: estrategias terapéuticas, incorporación de fármacos, guías clínicas y momento de inicio de TAR. 
Salud Metropolitano Centro, en el Hospital Clínico San Borja Arriarán, denominado Fundación Arriarán e inaugurado en 1991, fue el primer centro de atención en el sistema público nacional y se mantiene como el mayor del país, llegando a una población total atendida de más de 6.500 adultos a fines de 2017. El centro, su equipo profesional y la población en atención han vivido los hitos de manejo mencionados. Luego de 25 años de funcionamiento se hace necesario hacer una evaluación de los resultados del programa.

\section{Objetivo}

Evaluar la mortalidad, retención, abandono y traslado de pacientes adultos con infección por VIH ingresados entre los años 1990 y 2014 en un centro de referencia de atención $\mathrm{VIH}$, de acuerdo con las distintas etapas relevantes del programa nacional de atención integral de VIH/SIDA y la evolución general del manejo de la epidemia. Se cuantificó la mortalidad cruda de la población y no fue un objetivo analizar las causas de muerte ni tampoco su evolución clínica o de laboratorio.

\section{Pacientes y Métodos}

Estudio retrospectivo de análisis de bases de datos actualizadas de pacientes en control en Fundación Arriarán (FA).

Se evaluó según año de ingreso la mortalidad, abandono, traslado o retención en control clínico de pacientes a 1, 3, 5, 7 y 10 años, así como el estado general de toda la población atendida respecto a esos parámetros al 31 de diciembre de 2015.

Los períodos fueron ordenados según hitos relevantes ocurridos a nivel nacional en el manejo clínico de los pacientes con infección por VIH y corresponden a los siguientes:

Período A: PreTAR (1990-1992). Ausencia de antirretrovirales.

Período B: Monoterapia (1993-1995). Disponibilidad de zidovudina.

Período C: Biterapia (1996-1998). Disponibilidad de inhibidores nucleósicos/nucleótidos de transcriptasa reversa usados en combinación.

Período D: Bi y triterapia (1999-2000). Coexistencia de regímenes de 2 y 3 antirretrovirales combinados.

Período E: TAR antigua, cobertura incompleta (2001-2002). Uso exclusivo de triterapia, con algunas drogas actualmente en desuso.
Período F: TAR antigua, cobertura completa (2003-2008). Cobertura de 100\% de la población con indicación de TAR según las guías vigentes durante este período.

Período G: TAR contemporánea (2009-2014). Cobertura de $100 \%$ de la población con medicamentos contemporáneos, incluyendo inhibidores de integrasa.

Se definió abandono como una ausencia de controles médicos o retiro de medicamentos por un plazo superior a 6 meses. Traslado fue definido como los pacientes que solicitaron un cambio en su centro de atención, siendo transferidos a otras unidades de atención pública.

Se consideraron como pacientes activos o en retención, todos quienes asistieron al menos una vez a control médico o retiro de medicamentos durante un período de 6 meses.

Se incluyeron los pacientes que reingresaron post abandono o traslado de acuerdo con el estado en el período de reingreso. Se estudiaron todos los casos de abandono sin reingreso para determinar su estado vital y se reportaron los fallecimientos, con fecha asignada según momento de ocurrencia, de acuerdo a la base de datos de acceso libre del Fondo Nacional de Salud (FONASA) y Registro Civil. Se consideró TAR antigua a los regímenes que incluían antirretrovirales actualmente en desuso.

El estudio fue aprobado por el Comité Ético Científico del Servicio de Salud Metropolitano Central en el marco de estudios poblacionales de cooperación epidemiológica internacional y se otorgó la dispensa de consentimiento por ser de manejo confidencial, anonimización de la información y con implicancias en la implementación de políticas públicas de salud.

\section{Resultados}

La población estudiada fue de 5.080 adultos. Se incluyeron pacientes ingresados desde el 01 de enero de 1990 al 31 de diciembre de 2014.

\section{Mortalidad}

La mortalidad a 1, 5 y 10 años fue de $40 \%$, $62 \%$ y $71 \%$ para el período A; $28 \%, 44 \%$ y $47 \%$ para el C; $10 \%, 13 \%$ y $16 \%$ para el E y de $2 \%$ y $7 \%$ a 1 y 5 años para el grupo $\mathrm{G}$, respectivamente. Las causas de muerte no fueron incorporadas en este análisis. 
En la Tabla 1, se pueden ver todos los períodos detallados.

La mortalidad global, durante el todo el período de observación hasta el 31 de diciembre de 2015, fue de 1.119 pacientes, correspondiente a $22 \%$ del universo total de pacientes. El $81 \%$ de estas muertes ocurrió antes del 2003, cuando aún los tratamientos antirretrovirales con tres drogas no tenían cobertura universal, con $49 \%$ de letalidad en ese grupo al final del período de observación.

Las tasas de mortalidad mostraron una ligera disminución en los períodos de indicación de inicio de TAR más precoz, según las guías clínicas oficiales del MINSAL disponibles a esos respectivos períodos. Cuando el inicio de TAR en pacientes asintomáticos se realizaba con un recuento de linfocitos CD4 < 200 céls $/ \mathrm{mm}^{3}$ (1993-2008), la mortalidad fue de $14 \%, 20 \%$ y $23 \%$ comparado con el inicio de TAR con CD4 $<350$ céls $/ \mathrm{mm}^{3}$ (2009-2012) de 3\%, 5\% y 7\% al año 1, 3 y 5 de seguimiento, respectivamente. Cabe destacar que con inicio de TAR en pacientes con recuento de CD4 basal menor a $<500$ céls $/ \mathrm{mm}^{3}$, la mortalidad al año ha sido de sólo $1 \%$.

Tabla 1. Mortalidad a 1, 3, 5, 7 y 10 años desde el inicio de TAR según período de observación

\begin{tabular}{|ccccccc|}
\hline & & \multicolumn{5}{c|}{ Mortalidad (\%) } \\
\hline & Año & $\mathbf{1}$ & $\mathbf{3}$ & $\mathbf{5}$ & $\mathbf{7}$ & $\mathbf{1 0}$ \\
\hline A & $(330)$ & 40 & 48 & 62 & 67 & 71 \\
\hline B & $(397)$ & 31 & 50 & 57 & 60 & 63 \\
\hline C & $(485)$ & 28 & 42 & 44 & 46 & 47 \\
\hline D & $(303)$ & 13 & 18 & 19 & 21 & 22 \\
\hline E & $(348)$ & 10 & 11 & 13 & 15 & 16 \\
\hline F & $(1.255)$ & 4 & 6 & 8 & 9 & \\
\hline G & $(1.962)$ & 2 & 6 & 7 & & \\
\hline
\end{tabular}

Los valores en gris incluyen sólo a la proporción de pacientes que alcanzó a completar el período.

Período A: (1990-1992) preTAR.

Período B: (1993-1995) monoterapia.

Período C: (1996-1998) biterapia.

Período D: (1999-2000) bi y triterapia.

Período E: (2001-2002) TAR antigua, cobertura incompleta.

Período F: (2003-2008) TAR antigua, cobertura completa.

Período G: (2009-2014) TAR contemporánea.

\section{Abandono}

La tasa de abandono fue fluctuante entre los grupos y varió a final del año 1 entre $4 \%(\mathrm{G})$ y $12 \%$ (D) con promedio de 7\%. A los 5 años de observación la tasa de abandono osciló entre $9 \%$ (E) y $19 \%$ (B), con un promedio 14\%, y a los 10 años entre $12 \%$ (E) y $22 \%$ (B), con un promedio de $17 \%$ respectivamente (excluido período 2009-2014).

En la Tabla 2 se observan los períodos detallados.

$\mathrm{Al} 31$ de diciembre de 2015, del total de pacientes observados durante los 25 años de seguimiento, cumplían criterios de abandono, 608 pacientes, correspondiente a $11 \%$.

\section{Retención}

A 10 años de haber iniciado controles, sólo $22 \%$ de los ingresados previo a la disponibilidad de TAR (1990-1998) estaba activo, luego de contar con una cobertura universal de TAR (2003) la población activa a 10 años fue siempre mayor o igual a $66 \%$.

En la Tabla 3 se observan todos los períodos detallados.

Al 31 de diciembre de 2015, 60\% de toda la

Tabla 2. Abandono a 1, 3, 5, 7 y 10 años desde el inicio de TAR según período de observación

\begin{tabular}{|ccrrrrr|}
\hline & \multicolumn{7}{c|}{ Abandono (\%) } \\
& Año & $\mathbf{1}$ & $\mathbf{3}$ & $\mathbf{5}$ & $\mathbf{7}$ & $\mathbf{1 0}$ \\
\hline & $\mathbf{n}$ & & & & & \\
\hline A & $(330)$ & 5 & 7 & 10 & 12 & 10 \\
\hline B & $(397)$ & 10 & 15 & 19 & 22 & 9 \\
\hline C & $(485)$ & 9 & 14 & 15 & 15 & 12 \\
\hline D & $(303)$ & 12 & 15 & 17 & 19 & 20 \\
\hline E & $(348)$ & 6 & 8 & 9 & 11 & 12 \\
\hline F & $(1.255)$ & 6 & 9 & 12 & 14 & \\
\hline G & $(1.962)$ & 4 & 7 & 10 & & \\
\hline
\end{tabular}

Los valores en gris incluyen sólo a la proporción de pacientes que alcanzó a completar el período.

Período A: (1990-1992) preTAR.

Período B: (1993-1995) monoterapia.

Período C: (1996-1998) biterapia.

Período D: (1999-2000) bi y triterapia.

Período E: (2001-2002) TAR antigua, cobertura incompleta. Período F: (2003-2008) TAR antigua, cobertura completa.

Período G: (2009-2014) TAR contemporánea. 
Tabla 3. Retención de pacientes en control activo a 1, 3, 5, 7 y 10 años desde el inicio de TAR según período de observación

\begin{tabular}{|ccccccc|}
\hline & \multicolumn{6}{c|}{ Retención (\%) } \\
\hline & Año & $\mathbf{1}$ & $\mathbf{3}$ & $\mathbf{5}$ & $\mathbf{7}$ & $\mathbf{1 0}$ \\
\hline & $\mathbf{n}$ & & & & & \\
\hline B & $(330)$ & 55 & 45 & 28 & 20 & 14 \\
\hline C & $(397)$ & 59 & 35 & 23 & 17 & 14 \\
\hline D & $(385)$ & 63 & 43 & 39 & 37 & 35 \\
\hline E & $(348)$ & 83 & 78 & 75 & 70 & 65 \\
\hline F & $(1.255)$ & 88 & 82 & 75 & 71 & \\
\hline G & $(1.962)$ & 92 & 83 & 77 & & \\
\hline
\end{tabular}

Los valores en gris incluyen sólo a la proporción de pacientes que alcanzó a completar el período.

Período A: (1990-1992) preTAR.

Período B: (1993-1995) monoterapia.

Período C: (1996-1998) biterapia.

Período D: (1999-2000) bi y triterapia.

Período E: (2001-2002) TAR antigua, cobertura incompleta. Período F: (2003-2008) TAR antigua, cobertura completa.

Período G: (2009-2014) TAR contemporánea. población ingresada entre 1990-2014 se mantenía en control activo en el centro y $6 \%$ había solicitado traslado. Un diagrama que resume la evolución al año 1,5 y 10 de admisión se ve en las Figuras $2 \mathrm{a}, 2 \mathrm{~b}, 2 \mathrm{c}$.

\section{Discusión}

Este estudio caracteriza la evolución de desenlaces mayores de una población adulta infectada por VIH atendida en un centro especializado del sistema público de salud en Chile, durante un período de 25 años, en un contexto en que se mantuvo el inicio de TAR en etapas avanzadas de la enfermedad. Este ingreso tardío ha mostrado una significativa disminución en el tiempo, destacando que las guías terapéuticas nacionales siempre han contemplado el inicio de terapia en etapas avanzadas (estado clínico de SIDA o recuentos de CD4 $<200$ céls $\left./ \mathrm{mm}^{3}\right)^{20}$, lo que en la práctica implicó que toda la población que iba cumpliendo con los requisitos vigentes iba iniciando tratamiento según la disponibilidad terapéutica.

Por otra parte, durante este largo período se ha ido produciendo una modificación del contexto

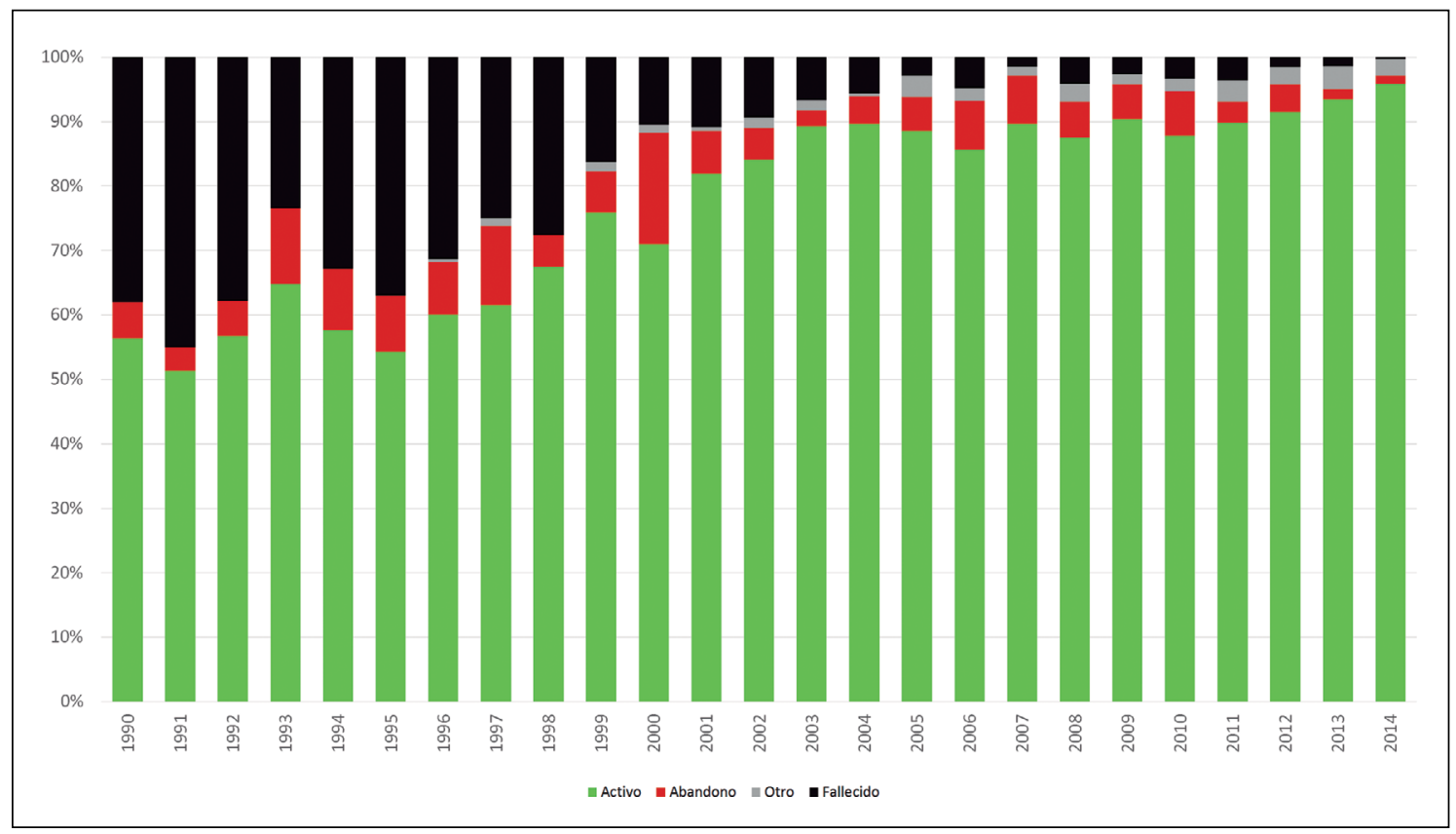

Figura 2a. Estado a 1 año de seguimiento por año de ingreso a control (1990-2014). 


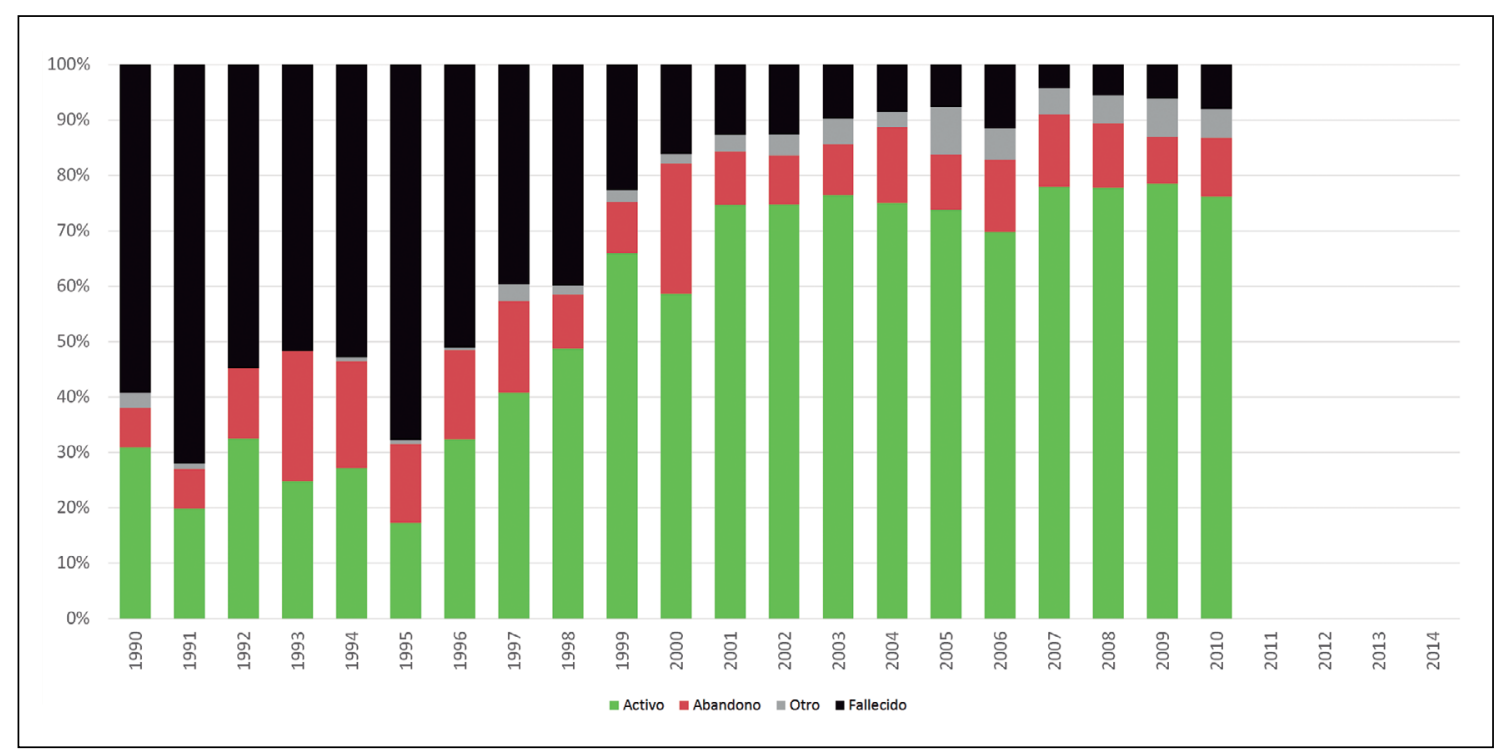

Figura 2b. Estado a 5 años de seguimiento según año de ingreso a control (1990-2014).

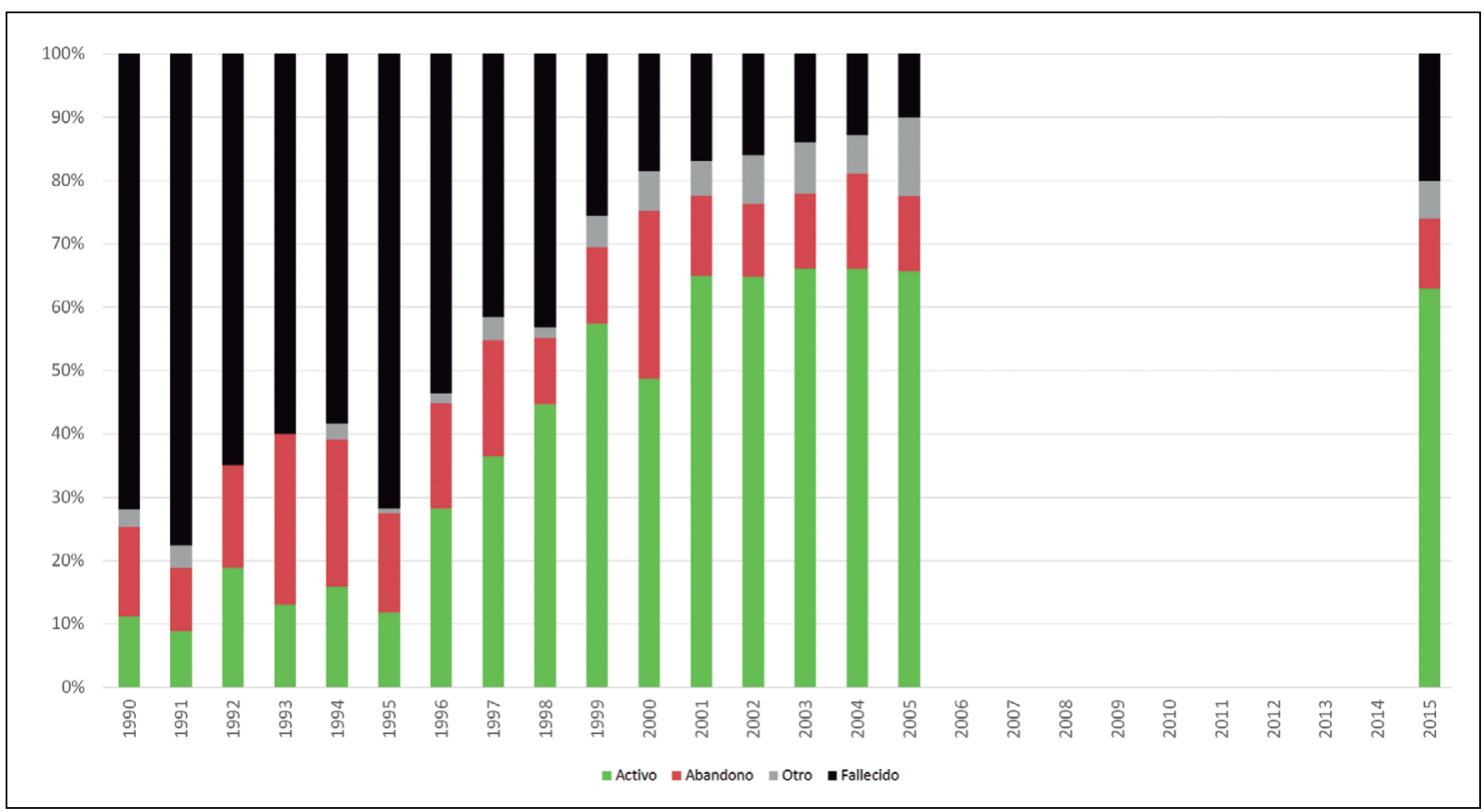

Figura 2c. Estados a 10 años de seguimiento según año de ingreso a control (se excluyen los períodos con menor duración de observación) y estado de toda la población al 31 de diciembre de 2015.

externo caracterizado por grandes avances en el conocimiento de la enfermedad, aparición de nuevas herramientas diagnósticas y terapéuticas a nivel global. A nivel nacional se ha asistido a un progresivo compromiso hacia la atención de la población afectada, con importantes avances en creación de centros de atención, disponibilidad de medicamentos, métodos de monitoreo y capacitación del recurso humano, así como la entrega de garantías entregadas por el GES ${ }^{17,21-28}$. Esto se 
ha traducido en una mejoría de la sobrevida y una significativa recuperación clínica en la población del sistema público de salud, incluso antes de la incorporación de la $\mathrm{TAR}^{27}$. A nivel nacional también ha sido reportada la respuesta clínica y virológica en pacientes pertenecientes mayoritariamente al sistema de salud privado ${ }^{29}$.

Este esfuerzo nacional, descrito para un centro -Fundación Arriarán- pero muy representativo del programa a nivel nacional, ha resultado en una espectacular modificación de la historia natural de la enfermedad en la población infectada que accede a atención especializada y tratamiento específico, particularmente respecto a mortalidad, la que al primer año de control en la era previa a la terapia era de $40 \%$ comparado con la actual $1 \%$ o menos; en donde, además, sólo una muy baja fracción de los pacientes en control no se encuentra recibiendo tratamiento. Destaca adicionalmente que, del total de fallecimientos, $(22 \%$ de la población atendida entre 1990 y 2014), 4/5 partes ocurrieron en la primera mitad del extenso período de seguimiento del programa, cuando no había terapia adecuada disponible para la totalidad de población en control. El objetivo primario del estudio fue cuantificar la mortalidad cruda en el tiempo y relacionarla con los hitos y recomendaciones terapéuticos pues ha sido evidente que la alta mortalidad previa a la TAR era mayoritariamente debido a patologías asociadas a la infección por VIH (eventos definitorios de SIDA) y la implementación de terapia ha traído gran disminución de mortalidad. Lo que es también claro es que las causas de muertes han ido variando con un aumento en el tiempo de cánceres no asociados a SIDA y patologías crónicas no infecciosas ${ }^{30-32}$, siguiendo la misma tendencia que a nivel mundial.

El progresivo aumento de sobrevida es marcado, en cada una de las etapas en que se dividió el seguimiento y actualmente alcanza los estándares de países desarrollados ${ }^{33,34}$. El abandono a los controles $y$, por ende, a la terapia, no ha tenido una disminución tan significativa como la observada en mortalidad y sigue siendo un problema no resuelto. Es interesante destacar que durante este estudio se procedió a una búsqueda y seguimiento de los pacientes inicialmente catalogados como en abandono, pesquisando que algunos habían fallecido y que muchos sobrevivientes habían reingresado, se habían trasladado y que también había genuinos abandonos que no registraban controles en el sistema público ni contaban con datos disponibles (información obtenida, pero no incorporados en este reporte excepto para los datos de mortalidad). Con todo lo expuesto, 11\% de abandonos registrados es altamente representativo de la realidad y es un porcentaje elevado e insatisfactorio, considerando que a diferencia de otras enfermedades en las que retomando la terapia abandonada se recuperan los beneficios, en la infección por VIH el abandono se asocia a pérdida de eficacia de la TAR previamente en uso y surge la necesidad de regímenes terapéuticos más complejos (y costosos), sin mencionar el aumento de la morbilidad y una reaparición de la transmisibilidad, incluso con la posibilidad de traspaso de cepas resistentes ${ }^{35,36}$.

Una proporción baja de los pacientes, $6 \%$, fue registrada como traslado dentro del sistema público, lo que muestra la coordinación y funcionamiento en red del programa. Con todo, es destacable que cerca de $2 / 3$ del total de pacientes se hayan mantenido en control activo al final de la evaluación. Cabe mencionar que, al hacer el análisis en relación con la oportunidad de inicio de tratamiento de acuerdo a las guías nacionales, se observó una consistente disminución de mortalidad en los períodos en que el inicio de TAR se indicaba en etapas más precoces, con recuento de linfocitos CD4 mayores. Esto se encuentra en concordancia con la experiencia cooperativa internacional -el estudio START- en la que participó este centro, que mostró inequívocamente mejores resultados de sobrevida y morbilidad con inicios de tratamiento precoces, incluso en aquellos con mínima o nula inmunosupresión ${ }^{8}$.

El estudio tiene limitaciones, entre las que destaca que no se correlacionó el estado clínico ni inmunológico al ingreso, ni al inicio de TAR con los desenlaces, así como tampoco con los resultados clínicos y virológicos de las distintas terapias ofrecidas; pero esto no invalida los resultados principales del estudio: una drástica reducción de la mortalidad. Una aproximación a los resultados virológicos e inmunológicos se puede extraer de un estudio cooperativo internacional en el que participó este centro, de seguimiento por 10 años de una población latinoamericana que inició TAR, antes de 2005, en la que la población de pacientes mostraba una mortalidad de 13,6\%; abandono de $24,2 \%$, indetectabilidad viral plasmática de $85 \%$ en la población activa e incremento de linfocitos 
CD4 de una mediana basal de 153 a 517 cels $/ \mathrm{mm}^{3}$ al final del período del estudio ${ }^{37}$.

En suma, el programa nacional de atención integral del VIH/SIDA y acceso expandido a TAR, evaluado en este centro, ha mostrado que el esfuerzo ha valido la pena y que se han salvado miles de vidas y evitado miles de nuevas infecciones por la supresión viral obtenida. Creemos que estos resultados, son representativos de lo logrado en el resto de los centros públicos de atención de VIH/SIDA en el país. La aplicación oficial de la recomendación de la OMS de proveer terapia inmediata a todos los infectados, independiente de su estado inmunológico y clínico, puede optimizar aún más estos buenos resultados. Paralelamente, la OMS ha lanzado la estrategia 90-90-90: la cual consiste en que $90 \%$ de los infectados conozcan su diagnóstico, $90 \%$ de estos se encuentren en tratamiento y $90 \%$ de los tratados estén en éxito virológico ${ }^{38}$. El país parece estar bien preparado y cumpliendo o cerca de cumplir los dos últimos componentes de esta estrategia. Un mayor compromiso para disminuir la brecha entre población infectada y la diagnosticada, para incorporar ésta en las siguientes etapas de la cascada de atención, tanto a través de políticas públicas de salud como por iniciativa de los prestadores individuales de salud, sin duda logrará acercarse a la ambiciosa, pero realista meta de la OMS. Creemos que el país puede y debe hacerlo.

Agradecimientos. El estudio no se habría podido realizar sin el trabajo y estímulo de todo el personal de Fundación Arriarán a lo largo del extenso período de funcionamiento, así como de los miles de pacientes que han confiado su salud a las instituciones del Servicio de Salud Metropolitano Central.

Se agradece la contribución de CCASAnet (Caribbean, Central and South America network for HIV epidemiology, miembro de la International Epidemiologic Databases to Evaluate AIDS (leDEA) financiada por el National Institute of Health, EE. UU., (U01AI069923).

Se agradece al Departamento Programa Nacional de Prevención y Control del VIH/SIDA e ITS, DIPRECE/Subsecretaría de Salud Pública, Ministerio de Salud, Chile, por la información epidemiológica y operacional entregada.

Parte de este trabajo fue presentado en el XXXV Congreso Chileno de Infectología, 13 al 15 de octubre de 2016, Valdivia, en donde recibió el premio Sociedad Chilena de Infectología al mejor trabajo del congreso.

\section{Referencias}

1. Moore RD, Keruly JC, Gebo KA, Lucas GM. An improvement in virologic response to highly active antiretroviral therapy in clinical practice from 1996 through 2002. J Acquir Immune Defic Syndr 2005; 39 (2): 195-8.

2. Palella FJ Jr, Baker RK, Moorman AC, Chmiel JS, Wood KC, Brooks JT, et al. HIV Outpatient Study Investigators. Mortality in the highly active antiretroviral therapy era: changing causes of death and disease in the HIV outpatient study. J Acquir Immune Defic Syndr 2006; 43 (1): 27-34.

3. Moore RD, Keruly JC, Bartlett JG. Improvement in the health of HIV-infected persons in care: reducing disparities. Clin Infect Dis 2012; 55 (9): 1242-51.

4. Fox MP, Rosen S. Retention of Adult Patients on Antiretroviral Therapy in Low- and Middle-Income Countries: Systematic Review and Meta-analysis 2008-2013. J Acquir Immune Defic Syndr 2015; 69 (1): 98-108.

5. Kleeberger CA, Buechner J, Palella F, Detels R, Riddler S, Godfrey R, et al. Changes in adherence to highly active antiretroviral therapy medications in the Multicenter AIDS Cohort Study. AIDS 2004; 18 (4): 683-8.

6. Carriquiry G, Fink V, Koethe JR, Giganti MJ, Jayathilake K, Blevins M, et al. Mortality and loss to follow-up among HIV-infected persons on long-term antiretroviral therapy in Latin America and the Caribbean. J Int AIDS Soc 2015; 10; 18: 20016.

7. Wolff MJ, Cortés CP, Shepherd BE, Beltrán CJ. Chilean AIDS Cohort Study Group. Long-term outcomes of a national expanded access program to antiretroviral therapy: the Chilean AIDS cohort. J Acquir Immune Defic Syndr 2010; 55 (3): 368-74.

8. The INSIGHT START Study Group. Initiation of Antiretroviral Therapy in Early Asymptomatic HIV Infection. N Engl J Med 2015; 373: 795-807.

9. Cotte L, Ferry T, Pugliese P, Valantin MA, Allavena C, Cabié A, et al. Dat'AIDS study group. Effectiveness and tolerance of single tablet versus once daily multiple tablet regimens as first-line antiretroviral therapy - Results from a large french multicenter cohort study. PLoS One 2017; 12 (2): e0170661.

10. Montaner JS, Lima VD, Harrigan PR, Lourenço L, Yip B, Nosyk B, et al. Expansion of HAART coverage is associated with sustained decreases in HIV/AIDS morbidity, mortality and HIV transmission: the "HIV Treatment as 
Prevention" experience in a Canadian setting. PLoS One 2014; 9 (2): e87872.

11. Ministerio de Hacienda. Evaluación del Programa de prevención y control del VIH/SIDA y las ETS. Comisión Nacional de SIDA, Informe final de evaluación 19902000. http://www.dipres.gob.cl/595/articles-14900_doc_ pdf (Accedido el 30 de marzo de 2017).

12. Ley 19.779 establece normas relativas al Virus de Inmuno Deficiencia Humana y crea bonificación fiscal para enfermedades catastróficas. Publicada en Diario Oficial 14 de diciembre de 2001. http://www.diariooficial.interior.gob.cl/media/2001/12/14/do-20011214.pdf

13. Decreto $\mathrm{N}^{\circ} 228$ de 2005 del Ministerio de Salud y Ministerio de Hacienda, publicado en el Diario Oficial el 30 de enero de 2006. http://bcn.cl/22sfr

14. Ministerio de Salud. Guía Clínica para la atención de personas adultas que viven con VIH/SIDA, octubre de 2001. http://www.criaps.cl/descarga/doc_elect_guia_clinica_conasida.pdf (Accedido el 30 de marzo de 2017).

15. Ministerio de Salud. Guía Clínica Síndrome de la Inmunodeficiencia Adquirida. VIH/SIDA. 1st Ed. Santiago: Minsal, 2005. http://www.paho.org/hq/dmdocuments/2010/Chile\%20ADULTOS.GESTANTES. NINOS.2005.pdf.

16. Ministerio de Salud. Guía Clínica Síndrome de Inmunodeficiencia Adquirida VIH/SIDA. Santiago: MINSAL, 2010. ISBN: 978-956-8823-87-0http://www. bibliotecaminsal.cl/wp/wp-content/uploads/2016/01/ chile_sida-guia.pdf

17. Ministerio de Salud. Guía Clínica AUGE Síndrome de la Inmunodeficiencia Adquirida VIH/SIDA, Santiago MINSAL 2013. http://web.minsal.cl/sites/default/files/ files/GPCVIH.pdf

18. WHO Guidelines Approved by the Guidelines Review Committee. Guideline on When to Start Antiretroviral Therapy and on Pre-Exposure Prophylaxis for HIV. Geneva: World Health Organization; 2015 Sep. https:// www.ncbi.nlm.nih.gov/books/NBK327115/ (Accedido el 30 de marzo de 2017).

19. WHO. Consolidated guidelines on the use of antiretroviral drugs for treating and preventing HIV infection. Recommendations for a public health approach - Second edition, Geneva World Health Organization. Junio de 2016. http://www.who.int/hiv/pub/arv/arv-2016/en/\# (Accedido el 30 de marzo de 2017).

20. Beltrán C, Zitko P, Wolff M, Bernal F, Asenjo A, Fernández AM. Evolución de las características epidemiológicas y clínicas de pacientes adultos del programa nacional al inicio de la terapia anti-retroviral en la Cohorte Chilena de SIDA, 2001-2015. Rev Chilena Infectol 2016; 33 (S1) $2-10$.
21. Wolff M, Diomedi A, Morales O, Bidart T, Dabanch J, Bustamante, et al. Seguimiento prospectivo de una población infectada por VIH con y sin posibilidades de terapia anti-retroviral: impacto en sobrevida y complicaciones. Rev Med Chile 2001; 129 (8): 886-94.

22. Wolff M, Beltrán C. Programa de acceso ampliado a terapia anti retroviral en Chile, Plan Auge y Cohorte Chilena de SIDA: Tres iniciativas complementarias. Rev Chilena Infectol 2004; 21 (4): 281-4.

23. Wolff MJ, Beltrán CJ, Vásquez $\mathrm{P}$, Ayala MX, Valenzuela M, Berríos G, et al. The Chilean AIDS cohort: a model for evaluating the impact of an expanded access program to antiretroviral therapy in a middle-income country-organization and preliminary results. J Acquir Immune Defic Syndr 2005; 40 (5): 551-7.

24. Wolff M, Álvarez P, Flores I, Northland R, Wolff C. Evolución de mortalidad y estado actual de una población infectada por VIH controlada en un centro multiprofesional. Rev Med Chile 2006; 134: 581-8.

25. Cortés C, Beltrán C, Muñoz R, Daube E, Wolff M. por el Grupo SIDA Chile. Cohorte Chilena de SIDA. Impacto de la inmunodepresión basal y su grado de recuperación al año de terapia antirretroviral en sobrevida, complicaciones oportunistas y reacción de recuperación inmune. Rev Med Chile 2008; 136: 1503-10.

26. Wilson G, Wolff M. Una década de terapia anti-retroviral: Perfil de pacientes con 10 años de triterapia de alta efectividad. Rev Chilena Infectol 2012; 29 (3): 337-43.

27. Wolff MJ, Cortés CP, Shepherd BE, Beltrán CJ. Chilean AIDS Cohort Study Group. Long-term outcomes of a national expanded access program to antiretroviral therapy: the Chilean AIDS cohort. J Acquir Immune Defic Syndr 2010; 55 (3): 368-74.

28. Departamento de Epidemiología. División de Planificación Sanitaria, Ministerio de Salud de Chile. Informe Nacional: evolución de la infección por VIH/SIDA Chile 1984-2012. Rev Chilena Infectol 2015; 32 (S1): 17-43.

29. Ceballos ME, Rojas A, Donato $P$, Huilcamán M, Rivera $\mathrm{G}$, López T, et al. Respuesta virológica e inmunológica a la terapia anti-retroviral en pacientes portadores de infección por VIH atendidos en una red de salud académica de Chile. Rev Chilena Infectol 2016; 33 (5): 531-6.

30. Smith CJ, Ryom L, Weber R, Morlat P, Pradier C, Reiss $\mathrm{P}$, et al for the D:A:D Study group. Trends in underlying causes of death in people with HIV from 1999 to 2011 (D: A:D): a multicohort collaboration. Lancet 2014; 384 (9939): 241-8.

31. Farahani M, Mulinder H, Farahani A, Marlink R. Prevalence and distribution of non-AIDS causes of death among HIV-infected individuals receiving antiretroviral 
therapy: a systematic review and meta-analysis. Int J STD AIDS 2017; 28 (7): 636-50.

32. Engels EA, Yanik EL, Wheeler W, Gill MJ3 Shiels MS, Dubrow R, et al. For the North American AIDS Cohort Collaboration on Research and Design of the International Epidemiologic Databases to Evaluate AIDS. Cancer-Attributable Mortality Among People with Treated Human Immunodeficiency Virus Infection in North America. Clin Infect Dis 2017; 65 (4): 636-43.

33. Althoff KN, Rebeiro PF, Hanna DB, Padgett D, Horberg MA, et al. North American Aids Cohort Collaboration on Research and Design (NA-ACCORD) and Caribbean, Central and South America Network for Hiv Epidemiology (CCASAnet). A picture is worth a thousand words: maps of HIV indicators to inform research, programs, and policy from NA-ACCORD and CCASAnet clinical cohorts. JInt AIDS Soc 2016; 19 (1): 20707.

34. Althoff KN, Buchacz K, Hall HI, Zhang J, Hanna DB, Rebeiro P, et al. North American AIDS Cohort Collaboration on Research and Design U.S. trends in antiretroviral therapy use, HIV RNA plasma viral loads, and CD4
T-lymphocyte cell counts among HIV-infected persons, 2000 to 2008. Ann Intern Med 2012; 157 (5): 325-35.

35. Hofstra LM, Sánchez Rivas E, Nijhuis M, Bank LE, Wilkinson E, Kelly K, et al. High Rates of Transmission of Drug-resistant HIV in Aruba Resulting in Reduced Susceptibility to the WHO Recommended First-line Regimen in Nearly Half of Newly Diagnosed HIV-infected Patients. Clin Infect Dis 2017; 64 (8): 1092-7.

36. Lepik KJ, Harrigan PR, Yip B, Wang L, Robbins MA, Zhang WW, et al. Emergent drug resistance with integrase strand transfer inhibitor-based regimens. AIDS 2017; 31 (10): 1425-34.

37. Wolff MJ, Giganti MJ, Cortés CP, Cahn P, Grinsztejn B, Pape JW, et al. A decade of HAART in Latin America: Long term outcomes among the first wave of HIV patients to receive combination therapy. PLoSOne 2017; 12 (6): e0179769.

38. UNAIDS. Geneva: UNAIDS; 2014. 90-90-90: an ambitious treatment target to help end the AIDS epidemic. http://www.unaids.org/sites/default/files/media_asset/90-90-90_en_0.pdf (Accedido el 4 de abril de 2017). 